\title{
Violencia conyugal y productividad en mujeres del Municipio de Durango, Durango, México
}

\section{Domestic violence and women's productivity in the municipality of Durango, Durango, Mexico}

\author{
Sandra Mancinas / José ZúNiga \\ Sandra Mancinas. Profesora-Investigadora de la Facultad de Trabajo Social y Desarrollo Humano de la Universidad Autónoma de \\ Nuevo León, México.E-mail: sandramancinas@hotmail.com \\ José Zúñiga. Profesor-Investigador de la Facultad de Trabajo Social y Desarrollo Humano de la Universidad Autónoma de Nuevo \\ León, México. Dirección Postal Facultad de Trabajo Social y Desarrollo Humano, División de Estudios de Posgrado Universidad \\ Autónoma de Nuevo León Cd. Universitaria s/n San Nicolás de los Garza, Nuevo León. C.P.66451
}

\begin{abstract}
Resumen
Se realizó un estudio trasversal, ex post-facto, en el municipio de Durango, Durango, México. La unidad de análisis estuvo constituida por 120 mujeres casadas o unidas, que desempeñaban trabajo asalariado y vivian violencia conyugal. El propósito central fue indagar en qué forma repercute la violencia conyugal en su productividad. La productividad incluyó el estudio de nueve variables dependientes: salud reportada, salud objetiva, estrés, número de incapacidades laborales, número de horas trabajadas por semana, ausentismo, motivación, satisfacción laboral y permanencia en el empleo. Se consideraron cuatro tipos de violencia conyugal como variables independientes: violencia conyugal física, sexual, emocional y económica. Se observó que la violencia conyugal emocional y la sexual son los dos tipos de violencia que más se asocian a elementos contraproductivos y/o los predicen.

Palabras claves. Violencia doméstica, productividad, género.

Abstract

The authors performed a transversal study, ex post facto, in the town of Durango, Durango, Mexico. The unit of analysis consisted of 120 women married or cohabiting, who held paid employment and lived domestic violence. The central purpose was to investigate how domestic violence affects their productivity. The productivity study included nine dependent variables: reported health, objective health, stress, number of work disabilities, number of hours worked per week, absenteeism, motivation, job satisfaction and job tenure. We considered four types of spousal abuse as independent variables: physical spousal abuse, sexual, emotional and economic. It was noted that emotional spousal violence and sex are two more types of violence associated with counterproductive elements and / or predict.
\end{abstract}

Key words. Domestic violence, productivity, gender.

\section{Introducción}

La violencia familiar se manifiesta en distintas formas y direcciones. El maltrato infantil, la violencia hacia los ancianos y la violencia entre cónyuges, son las rutas que a menudo sigue la violencia en la familia (Corsi, 1999). La violencia conyugal es una de las muchas formas de maltrato hacia la mujer. Este tipo de violencia incluye acciones y omisiones de "parte de la pareja con quien la mujer mantiene un vínculo de intimidad" (Dohmen, 1999: 65). El maltrato que sufre la mujer puede ser físico, emocional, sexual y económico (Dohmen, 1999; Torres, 2001).

Con frecuencia las mujeres receptoras de violencia se muestran con poca energía, preocupadas por problemas derivados de la violencia, por lo que ésta se convierte en un obstáculo para el logro de sus metas, disminuye sus habilidades y rendimiento laboral (Corsi, 1999; Sable y otros 1999; Carrillo, 1992). La violencia conyugal también dificulta el acceso y permanencia en el mercado laboral de las 
mujeres que la experimentan (Sable y otros, 1999; OPS y OMS, 2002).

Adicionales a los dolorosos efectos de la violencia conyugal en la subjetividad y en el cuerpo de las mujeres, se encuentran los costos económicos para los países, los cuales. Abarcan tanto el valor de los bienes y servicios utilizados para tratar o prevenir la violencia familiar, como el valor de los bienes y servicios no producidos cuando el maltrato conyugal lleva a un aumento del ausentismo, las incapacidades, las enfermedades y la pérdida del empleo de las mujeres asalariadas que viven violencia conyugal (Morrison y Orlando 1999: 49).

Un estudio realizado en la India observó que 13\% de las entrevistadas renunciaron a sus trabajos como consecuencia del ausentismo provocado por la violencia conyugal. Y en Estados Unidos, las mujeres agredidas por sus parejas eran más proclives a permanecer desempleadas o a la rotación de empleo (OPS, y OMS, 2002).

Estimaciones realizadas indican que las pérdidas económicas para Estados Unidos, como efectos de la violencia familiar -sólo en los rubros de salud y productividad-, se ubican entre los 5,000 y 10,000 millones de dólares anuales (Gelles, 1992, citado en Morrison y Orlando, 1999). Canadá por su parte gastó 3,200 millones de dólares americanos durante 1993 (Greaves, y otras 1995, citando en Morrison y Orlando, 1999).

La situación en América Latina, concretamente en Santiago de Chile y en Managua, no es distinta, un estudio indica que la violencia conyugal limitó el acceso al mercado laboral a $32.8 \%$ de las chilenas; además redujo su ingreso en 1.550 millones de dólares. Mientras que en Nicaragua, el ingreso de las mujeres como efecto derivado del mismo problema, se redujo en 29.5 millones de dólares (Morrison y Orlando, 1999).

Algunos estudios sugieren que la relación inversa entre la violencia conyugal y la productividad laboral es indirecta. La violencia conyugal impacta la salud, aumenta el ausentismo y las incapacidades laborales, disminuye el número de horas trabajadas, la satisfacción y la motivación laboral y estos elementos a su vez inciden de manera negativa en la productividad (Carrillo, 1992; Lambert, 2000; Heise, 1994; Sable, 1999; Corsi, 1999; Morrison y Orlando, 1999). Según Heise (1994: 32), las mujeres receptoras de violencia conyugal "no pueden brindar con plenitud su trabajo o sus ideas creativas mientras están agobiadas por las secuelas físicas y psicológicas del abuso." (Heise, 1994: 32).
En este sentido, se puede decir que los impactos de la violencia conyugal no sólo se perciben en la salud física y mental de las mujeres que la experimentan, sino que sus efectos se extienden a los sistemas económicos de los países. Por ello evidenciar la violencia conyugal -a través de la investigación- como un elemento extra-organizacional que afecta la productividad de la mujer trabajadora, puede ayudar a mostrar a evidenciar su impacto social.

El presente trabajo de investigación tuvo como propósitos centrales indagar en qué forma repercute la violencia conyugal en la productividad de mujeres asalariadas del municipio de Durango, Durango; además busca identificar cuál tipo de violencia conyugal (emocional, física, sexual y económica) tiene mayor impacto en la productividad de la población mencionada.

La hipótesis central asume la existencia de una relación inversa entre la violencia conyugal y la productividad de las mujeres asalariadas entrevistadas.

\section{Metodología}

Se usó un diseño transversal, no experimental o ex post-facto. Se empleó un muestreo no probabilístico con sujetos voluntarios. La muestra estuvo constituida por 120 mujeres que vivían violencia conyugal y asistieron durante los meses de enero y febrero de 2003 a la Dirección de Asistencia Jurídica y Procuraduría de la Defensa del Menor, la Mujer y la Familia; así como a las mesas del Ministerio Público 12 y 15 especializadas en Violencia Intrafamiliar del municipio de Durango, México. Las entrevistadas se encontraban en un rango de edad de 18 a 49 años, desempeñaban un trabajo asalariado formal y estaban casadas o unidas.

Se consideraron cuatro tipos de violencia conyugal como variables independientes: violencia física, emocional, sexual y económica. Se incluyeron también nueve variables dependientes: salud reportada, salud objetiva, estrés, número de incapacidades laborales, número de horas trabajadas por semana, ausentismo, motivación, satisfacción laboral y permanencia en el empleo.

Para recoger la información se elaboró una cédula de entrevista con 185 preguntas, 169 cerradas, precodificadas y 16 abiertas. Dicho instrumento de medición se dividió en tres grandes secciones: la primera de ellas para recopilar información sobre datos generales de las entrevistadas; la segunda sección incluyó preguntas sobre manifestaciones de violencia conyugal y la tercera parte indagó aspectos relacionados con la productividad de las 
entrevistadas. Para elaborar los ítems se examinaron las definiciones conceptuales de cada una de las variables dependientes e independientes, así como algunos instrumentos diseñados para otros estudios sobre familia y violencia familiar (Alvarado, 1997; Ribeiro, 1998; INEGI, 2000).

Una vez aprobada la cédula de entrevista (por un grupo de expertos), se realizó una prueba piloto con 35 casos para eliminar aquellas preguntas que resultaran confusas, así como para corregir posibles errores de redacción o de secuencia, que pudieran traducirse en problemas de validez y/o confiabilidad.

El procesamiento estadístico se realizó mediante el Statistical Package for Social Science (SPSS) versión 8.0 para Windows. El análisis descriptivo incluyó medidas de tendencia central (media) y distribución de frecuencias. Con el fin de indagar si existe una asociación estadísticamente significativa $(p<.05)$ entre los diferentes tipos de violencia conyugal y las variables de los elementos que afectan negativamente la productividad, se realizaron correlaciones. Siguiendo a Rubin y Babbie (1997), el coeficiente de correlación se escogió de acuerdo a los tipos de variables. Por ejemplo el coeficiente de Kendall's tau-b, se eligió cuando se trataba de variables ordinales-intervalo y viceversa, proporción-ordinal y viceversa. Se recurrió al coeficiente de Pearson, cuando se correlacionaron variables proporción-proporción, intervalo-intervalo, intervalo-proporción y viceversa.

\section{Análisis e interpretación de datos}

Los datos sociodemográficos indican que las entrevistadas tenían en promedio 32.67 años de edad. La media de los años de unión de la pareja fue de 11.10, mientras que el número de hijos osciló entre los 0 y 6 con una media de 2.57. El ingreso de las entrevistadas presentó una media de $2610.83^{1}$, con un rango de 800 a $9,000^{2}$ pesos mensuales. Siete de cada 10 mujeres entrevistadas manifestaron haber terminado estudios de secundaria o niveles educativos más altos.

Con relación a las características del trabajo que desempeñaban las entrevistadas, se observó que $35 \%$ de ellas prestaban sus servicios en el área de comercio y ventas, mientras que $25.8 \%$ realizaban trabajo de tipo técnico y sólo $10.8 \%$ desempeñaba un trabajo de tipo profesional. Los principales centros laborales a los que acudían las mujeres entrevistadas eran pequeños comercios (26.7\%), empresas privadas (25\%) y maquilas (15.8\%).

Como se ha mencionado antes, todas las mujeres entrevistadas vivían violencia conyugal, ello hace que las manifestaciones de ésta en términos porcentuales sean altas. Por ejemplo, los gritos (95\%), la mentira (93.3) y las prohibiciones (78.3\%) fueron las formas en que habitualmente su pareja les infligía violencia emocional.

Por su parte, la violencia sexual se ejercía a través de la celotipia (88.3\%), las relaciones sexuales forzadas (66.7\%) y la coerción hacia las mujeres para realizar actos sexuales que no deseaban (40.8\%). En tanto, los aventones (95\%), las bofetadas (75.8\%), los golpes contra la pared (56.7\%) y las patadas $(51.7 \%)$, fueron las manifestaciones de violencia conyugal física más observadas en las entrevistadas.

Finalmente, se observó que las formas más comunes de ejercer violencia económica eran la negación para cubrir necesidades recreativas $(77.3 \%)$ y educativas $(70.7 \%)$. La prohibición para trabajar fuera del hogar es un recurso que se emplea en 6 de cada 10 mujeres entrevistadas.

El análisis estadístico, tal como se muestra en el cuadro 1 , reveló que sí existe correlación entre la violencia conyugal y productividad. 
CUADRO No

CORRELACIONES ENTRE TIPOS DE VIOLENCIA CONYUGAL Y ELEMENTOS QUE AFECTAN LA PRODUCTIVIDAD

\begin{tabular}{|c|c|c|c|c|}
\hline & $\begin{array}{l}\text { Violencia } \\
\text { física }\end{array}$ & $\begin{array}{l}\text { Violencia } \\
\text { emocional }\end{array}$ & $\begin{array}{c}\text { Violencia } \\
\text { sexual }\end{array}$ & $\begin{array}{c}\text { Violencia } \\
\text { económica }\end{array}$ \\
\hline Satisfacción en el trabajo & --- & $-.189 * *$ & $-.145^{*}$ & -- \\
\hline Motivación laboral & -- & $-.216 * *$ & -- & -- \\
\hline Estrés & $.286^{* *}$ & $.507^{* *}$ & $.350 * *$ & $.263 * *$ \\
\hline $\mathrm{N}^{\circ}$ de horas trabajadas semanalmente & $.224 * *$ & $.170^{*}$ & -- & --- \\
\hline Inasistencia al centro de trabajo & --- & --- & $159 *$ & --- \\
\hline \multicolumn{5}{|l|}{ Permanencia en el empleo } \\
\hline Salud reportada & -- & -- & -- & -- \\
\hline Salud objetiva & --- & $.398 * *$ & $392 * *$ & $.185^{*}$ \\
\hline Incapacidad laboral & --- & --- & --- & --- \\
\hline
\end{tabular}

** Correlación significativa a nivel de p. $<0.01$

* Correlación significativa a nivel de p. $<0.05$

--- No hay correlación estadísticamente significativa (p>.05)

Los resultados de las correlaciones indican que a mayor violencia física, las mujeres entrevistadas viven mayor estrés. Esto coincide con los planteamientos de psicólogos laborales, como Lucas (1994) y Davis y Newstrom, (2000), quienes señalan que la dinámica familiar tiene efectos importantes en la vida laboral de las personas.

Al mismo tiempo, y aunque parezca contradictorio, se observó que a mayor violencia física, las entrevistadas trabajan más horas semanalmente. Pensamos que esto puede explicarse en la percepción subjetiva de las mujeres hacia al trabajo. Moreno (1999) considera que las mujeres buscan en el trabajo una compensación social que les ayude a sentirse útiles, ocupadas e independientes ${ }^{3}$. Por tanto, el lugar de trabajo es potencialmente un espacio de compensación emocional en el que las mujeres buscan sentirse auto-valoradas.

El hecho de que no se observaran asociaciones negativas entre la violencia conyugal física y el deterioro del estado de salud reportado y objetivo de las entrevistadas -aún cuando la relación entre ambas se encuentra ampliamente discutida y documentada (Lozano, 1999; Heise, 1994; Granados, 2002; Morrison y Orlando, 1999; OMS y OPS, 2002)- da paso a la importancia de las vivencias subjetivas de las entrevistadas. Granados (2002) y Heise (1994) encontraron que para algunas mujeres las humillaciones que acompañan la violencia física son más dolorosas que el golpe mismo. Así que es probable que las entrevistadas aunque hayan recibido violencia conyugal física no se asuman lastimadas físicamente, más bien relacionan las agresiones de este tipo como humillaciones, es decir como una forma de violencia emocional más que violencia física. Entonces, si estas mujeres no se sienten lastimadas físicamente, no acudirán a consulta para obtener una licencia médica; por ello no es extraño que el número de licencias de las entrevistadas no se encuentre asociado a la violencia conyugal física. Por otro lado, los resultados de la correlación entre la violencia conyugal emocional y los elementos contraproductivos revelaron que a mayor violencia emocional, menores son la satisfacción y la motivación laboral. Esta relación negativa ha sido documentada por distintos autores (Carrillo, 1992; Lambert, 2000; Heise, 1994; Sable, 1999; Corsi, 1999; Morrison y Orlando, 1999) quienes consideran que la violencia conyugal disminuye la motivación, la satisfacción y afecta la salud de las mujeres que la viven. Al igual que en la violencia física, el aumento de la violencia emocional trae

3. Por ejemplo, el $36.7 \%$ de las entrevistadas comentó que trabaja para sentirse útil y un $35 \%$ lo hace para ganar dinero y participar en los gastos del hogar. 
consigo mayor estrés en las mujeres entrevistadas. Después de la violencia emocional, la violencia sexual es el tipo de violencia que más se asocia a elementos que afectan negativamente la productividad. Al igual que en otros estudios (Heise, 1994; Morrison y Orlando, 1999), se encontró que cuando la violencia sexual aumenta las entrevistadas presentan mayor deterioro en su estado de salud objetiva, se sienten estresadas, menos satisfechas con su trabajo y aumentan sus inasistencias laborales. La relación negativa entre violencia y productividad se sostiene en el impacto de la primera en la subjetividad de las mujeres. La violencia es un evento que impacta su manera de ver el mundo porque obstaculiza el logro del estado positivo hacia las experiencias laborales, condición que según Locke, (1976, citado en Gómez 2000) es necesaria para la satisfacción laboral.

De la misma manera que Morrison y Orlando, (1999); Carrillo, (1992); Lambert, (2000); Heise, (1994) y Sable, (1999), en este trabajo se observó que la violencia conyugal está asociada al ausentismo laboral. Sin embargo, se observó que la relación entre ambas variables puede modificarse según el tipo de violencia conyugal de que se trate. Por ejemplo, de los cuatro tipos de violencia conyugal estudiados en el presente trabajo sólo la violencia sexual está asociada a los días laborales perdidos de las entrevistadas. Lo cuál no es extraño si se considera que este tipo de violencia es la de mayor impacto en la subjetividad femenina (Heise, 1994; OMS y OPS, 2002).

Con relación a la violencia conyugal económica, los resultados del análisis estadístico indicaron que cuando ésta aumenta, el nivel de estrés de las mujeres también lo hace, y su estado de salud se deteriora. El deterioro de salud objetivo de las entrevistadas ante el aumento de la violencia económica de que son receptoras, encuentra sentido en su definición. Para Torres (2001) este tipo de violencia se refiere al uso de los recursos materiales para transgredir los derechos de otra persona; sus manifestaciones abarcan la privación de los medios para satisfacer necesidades básicas, como las de salud (Torres, 2001). En el caso de las mujeres entrevistadas, $50.2 \%$ de ellas dijeron que alguna vez su pareja les han negado los medios para satisfacer necesidades de salud, lo que potencialmente se puede traducir en un deterioro de su estado de salud.

En resumen, esta investigación comprobó la existencia de una relación inversa entre la violencia conyugal y la productividad de las asalariadas entrevistadas. Las repercusiones de la violencia conyugal en la productividad se dan en diferentes formas e intensidades, según sea el tipo de violencia conyugal. En este estudio se observó que la violencia conyugal emocional, y la violencia conyugal sexual son los dos tipos de violencia que más se asocian a elementos contraproductivos.

Es importante subrayar que el estrés fue el elemento contraproductivo que más se asoció a los cuatro tipos de violencia conyugal y que la disminución de horas trabajadas, y la permanencia en el empleo, son dos variables que en la población estudiada parecen estar más asociadas a condiciones laborales y económicas, que a la violencia conyugal, sobre todo porque el desempleo constituye uno de sus principales problemas sociales del Estado de Durango, Durango México (Hernández, 2003).

Se concluye, que la violencia conyugal y sus efectos no deben ser considerados como algo monolítico, ya que la relación inversa entre ésta y la productividad adquiere singularidad de acuerdo al tipo de violencia, a la naturaleza de los elementos contraproductivos y al contexto económico, sociocultural y político en que las personas se encuentren inmersas.

\section{Referencias}

ALVARADO, G. y OTROS. (1998). Prevalencia de la violencia doméstica en la ciudad de Durango. Revista de Salud Pública en México. 40 (6) 481-486.

CARRILlO, R. (1992). La violencia contra la mujer, un obstáculo para el desarrollo. Nueva York: Fondo de las Naciones Unidas para la Mujer (UNIFEM).

CORSI, J. (1999). Una mirada abarcativa sobre el problema de la violencia intrafamiliar. En: Corsi, J. Violencia familiar. Una mirada interdisciplinaria sobre un grave problema social. Argentina: Ed. Paidós.15-63. Tercera reimpresión.

DAVIS, K. y NEWTROM J. (2000). Comportamiento humano en el trabajo. México: McGraw-Hill. Décima edición.

DOHMEN, M. (1999). Abordaje interdisciplinario del síndrome de la mujer maltratada. Proceso secuencial. En: Corsi, J. Violencia Familiar. Una Mirada Interdisciplinaria sobre un Grave Problema Social. Argentina: Ed. Paidós. 65-132.

GÓMEZ, A. y OTROS. (2000). Motivación y satisfacción laboral. En: Guillén, Carlos y Rocío Guil. Psicología del trabajo para relaciones laborales. España: Ed. Mc.GrawHill Interamericana de España.

GRANADOS, M. (2002). Salud Reproductiva y violencia contra la mujer. Un Análisis desde la Perspectiva de Genero. México. Secretaría de Salud. Gobierno del Estado de Nuevo León. 
HEISE, L. y OTRAS. (1994). Violencia contra la mujer: La carga oculta sobre la salud. Washington, D.C. Organización Panamericana de la Salud.

HERNÁNDEZ, E. (2003). Durangueñeidad. Durango: Instituto Municipal de Arte y Cultura.

INEGI. (2000). Violencia intramiliar: encuesta 1999. Aguascalientes, Ags: Instituto Nacional de Estadística, Geografía e Informática.

LAMBERT, LINDA C. (2000). Economic Context and Multiple Abuse Techniques. Violence Against Women (En línea), 6.(5). http://search.epnet.com.

LOZANO, R. (1999). Efectos de la violencia doméstica en la salud: Ciudad de México. En: Los costos del silencio. Violencia doméstica en las américas. Washington, D. C. Banco Interamericano de Desarrollo. 81-101

LUCAS, A. (1994). El estrés en el trabajo. En: Sociología de la empresa. Madrid: McGraw-Hill Interamericana de España. 56- 65.

MORENO, F. (1999). La violencia en la pareja. Revista Panamericana de Salud Pública, 5(4 y 5) 245-258.

MORRISON, A. y ORLANDO, M. (1999). El impacto socioeconómico de la violencia doméstica: Chile y Nicaragua. En: Los costos del silencio. Violencia doméstica en las américas. Washington, D. C. Banco Interamericano de Desarrollo. 49-80.

ORGANIZACIÓN PANAMERICANA DE LA SALUD y ORGANIZACIÓN MUNDIAL DE LA SALUD (2002). Informe mundial sobre la violencia y la salud. (En línea). http://www.paho.org/spanish/AM/PUB/capitulo_4.pdf (página consultada el 30 de octubre de 2003).

RIBEIRO, M. (2002). Ideología de género y transformación de los papeles conyugales en Monterrey, México. En: La familia en América del norte. Evolución, problemática y política. México: Trillas. 333-362.

RUBIN, A y BABBITE E. (1997). RESEARCH METHODS FOR Social Work. California: Brooks/Cole Publishin Company. (Third edition).

SABLE, M. y OTROS. (1999). Domestic violence among AFCD recipients: implications for welfare-to-work programs. Journal of Women and Social Work (en línea), 14 (2) http://search.epnet.com. Página consultada el 8 de noviembre de 2001.

TORRES, M. (2001). La Violencia en casa. México: Ed. Paidós. 